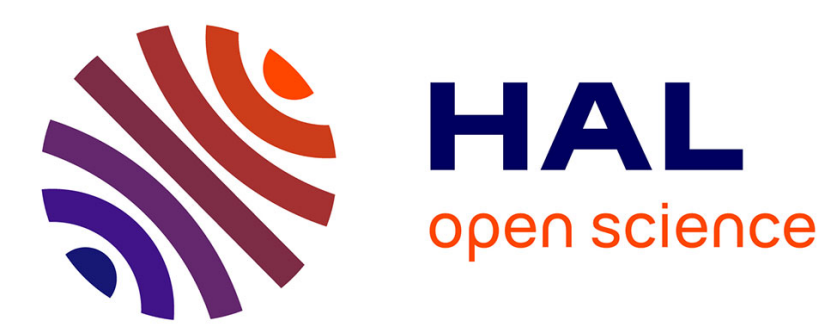

\title{
TAS and wind estimation from radar data
}

Daniel Delahaye, Stéphane Puechmorel

\section{To cite this version:}

Daniel Delahaye, Stéphane Puechmorel. TAS and wind estimation from radar data. DASC 2009, 28th Digital Avionics Systems Conference, Oct 2009, Orlando, United States. pp 2.B.5-1 - 2.B.5-16, 10.1109/DASC.2009.5347547 . hal-00938201

\section{HAL Id: hal-00938201 https://hal-enac.archives-ouvertes.fr/hal-00938201}

Submitted on 5 May 2014

HAL is a multi-disciplinary open access archive for the deposit and dissemination of scientific research documents, whether they are published or not. The documents may come from teaching and research institutions in France or abroad, or from public or private research centers.
L'archive ouverte pluridisciplinaire HAL, est destinée au dépôt et à la diffusion de documents scientifiques de niveau recherche, publiés ou non, émanant des établissements d'enseignement et de recherche français ou étrangers, des laboratoires publics ou privés. 


\title{
TAS AND WIND ESTIMATION FROM RADAR DATA
}

\author{
Daniel Delahaye ENAC 7, Ave Ed Belin 31055 Toulouse France \\ Stephane Puechmorel ENAC 7, Ave Ed Belin 31055 Toulouse France
}

\begin{abstract}
Accurate wind magnitude and direction estimation is essential for aircraft trajectory prediction. For instance, based on these data, one may compute entry and exit times from a sector or detect potential conflict between aircraft. Since the flight path has to be computed and updated on real time for such applications, wind information has to be available in real time too.The wind data which are currently available through meteorological service broadcast suffer from small measurement rate with respect to location and time. In this paper, a new wind estimation method based on radar track measures is proposed. When on board true air speed measures are available, a linear model is developed for which a Kalman filter is used to produce high quality wind estimate. When only aircraft position measures are available, an observability analysis shows that wind may be estimated only if trajectories have one or two turns depending of the number of aircraft located in a given area. Based on this observability conditions, closed forms of the wind has been developed for the one and two aircraft cases. By this mean, each aircraft can be seen as a wind sensor when it is turning. After performing evaluations in realistic frameworks, our approach is able to estimate the wind vectors accurately. Based on those local wind estimates, a global space-time wind field estimation using vector splines is interpolated in order to produce wind maps in the area of interest.The underline model for wind field computation is Shallow-Water, which assumes geostrophic wind. The accuracy of this wind map is dependent of the number wind estimates in a given zone. Further improvements to the estimation can be made by correlating with meteorological measurements.
\end{abstract}

\section{Introduction}

When an aircraft flies from a city A to a city B, it has to be managed by air traffic controllers in order to avoid collisions with others aircraft. Everyday, about 8000 aircraft fly in the French airspace, inducing a huge amount of control workload. Such a workload, is then spread by the mean of the airspace sectoring (the airspace is divided into geometrical sectors, each of them being assigned to a controller team). When a conflict between two (or more) aircraft is detected, the controller changes their routes (heading, speed, altitude) in order to keep a minimum distance between them during the crossing. All flying aircraft are then monitored during their navigation and so from departure till destination. The controller has a 2D visualization of his traffic by the mean of the radar screen. At the beginning of radar air traffic control, civil aviation authority used primary radar. In such a system, the radar send a high energy electromagnetic pulse (several mega watts (peak power) during one micro second) in the airspace waiting the echo back. This pulse propagates at the light speed $(c=300 \mathrm{~km}$ per second), touch the aircraft and comes back to the radar with a very low power (some nano watts). Based on the time difference between pulses, it is easy to compute the distance between the radar and the aircraft $\left(\Delta_{\text {distance }}=\frac{\Delta_{\text {time.c }}}{2}\right.$ where $c$ is the light speed $)$. This system gives a straight measure of the distance between the aircraft and the radar which is a mix between the $2 \mathrm{D}$ geographical distance and the altitude of the aircraft. When controllers used only primary radar, they had to asked the pilot to give his altitude and his ID in order to identify more precisely the spot they saw on their screens. The secondary radar really improved this system by the mean of active target concept. In this system, the aircraft is not passive anymore (from the radar point of view) but is equipped by an emitter which answers to the radar when the radar beam reaches the aircraft. Every time the aircraft is addressed by the radar, the aircraft emitter send back two codes called ModeA and ModeC. Those numbers are coded into octal and give respectively the ID and 
the altitude of the aircraft. When the radar receives back those two pieces of information, it is easy to compute the 2D geographical distance and the altitude of the aircraft. Another radar system, called Mode S, proposes to establish a data link between the radar and the aircraft during the time the aircraft is in the radar beam. This short connection enables to down link some flight data to the ground such like roll angle, true air speed, heading, altitude rate, etc....and enable to improve the radar tracking [13]. This communication between aircraft and the ground can be extended by the mean of satellites which are even accessible over the oceans where there is no radar.

All those radars deliver raw measures with additional noises coming from electromagnetic perturbations, electronic systems, etc.... Those measures are then filtered by the mean of a tracker filter in order to produce smooth trajectories.

When a controller observes its traffic on the radar screen, he tries to identify convergent aircraft which may be in conflict in a near future, in order to apply maneuvers that will separate them. The problem is to estimate where the aircraft will be located in this near future (5-10 minutes); this process is call trajectory prediction. This prediction may be also very useful in order to estimate the workload level in control sector to prevent over capacity event. As a mater of fact, it is very useful to estimate when an aircraft will enter a sector in order to compute the associated sector workload and to apply regulation if necessary. When a sector is expected to be overloaded, the aircraft involved in such a process will be speed up or slow down by the controller in order to adapt the demand to the actual capacity as much as possible. The trajectory prediction depends mainly on the residual noise after filtering (see [9]): the weight of the aircraft, the temperature and the wind. The residual noise is integrated with time with a growing covariance matrix indicating that the estimated position is less and less accurate. The weight of the aircraft is relevant in the flight dynamic model but is still a raw data. The engines of aircraft are sensitive to the air temperature and such a data is very useful to model the trust of the aircraft but it is also very difficult to measure on real time. Finally, the wind influences strongly the cinematic of the aircraft and limits also the trajectory prediction. Based on the available accuracy, the actual limit of the trajectory prediction is about 20 minutes. It means that after 20 minutes the uncertainty is so big that the estimated position is no more useful for any ATM purposes.

Several efforts have been tried to improved the trajectory prediction by estimating or suppressing the wind [15], [7], [16], [8], [4]. The presents paper, proposes a new method for estimating the wind around aircraft by the mean of observations of the radar tracks and some down linked data. One goal of this work is to show how it is possible to extract wind information from the radar observations.

The paper is organized as follow : the first part presents the relation between air speed vector, ground speed vector and the wind. The second part gives the observability condition of the wind based on the available measures. The third part present the Kalman filter and its non linear extensions. The fourth part presents the different models which have been used for the wind extraction. The fifth part presents some results and compares the performance of our models. Finally, the sixth part describes how a wind field map can be built using some spread measures of the wind.

\section{General Relations between Speed Vectors}

The following notations will be used in the paper. Vectors and matrices are shown with underlined symbols.

Speeds

$$
\underline{V}=\left[\begin{array}{l}
v_{x} \\
v_{y}
\end{array}\right] V=\sqrt{v_{x}^{2}+v_{y}^{2}} \theta_{g}=\arctan \left(\frac{v_{x}}{v_{y}}\right)
$$

Where $\theta_{g}$ is the The route angle (with reference to north). The same notation will be used for the air speed $\left(\underline{T}, t_{x}, t_{y}, \theta_{a}\right)$ and the wind $\left(\underline{W}, w_{x}, w_{y}, \theta_{w}\right)$. We have $: \underline{V}=\underline{T}+\underline{W}$. These relations are shown on figure 1.

\section{Air Turning Rate}

$$
\omega_{a}=\frac{d \theta_{a}(t)}{d t}
$$

\section{Ground Turning Rate}

$$
\begin{gathered}
\omega_{g}=\frac{d \theta_{g}(t)}{d t}=\frac{d[\arctan u(t)]}{d t}=\left(\frac{d[\arctan u]}{d u}\right) \cdot\left(\frac{d u}{d t}\right) \\
\text { with } u=\left(\frac{v_{x}}{v_{y}}\right) \\
\qquad \omega_{g}=\frac{\gamma_{x} \cdot v_{y}-v_{x} \cdot \gamma_{y}}{V^{2}}
\end{gathered}
$$




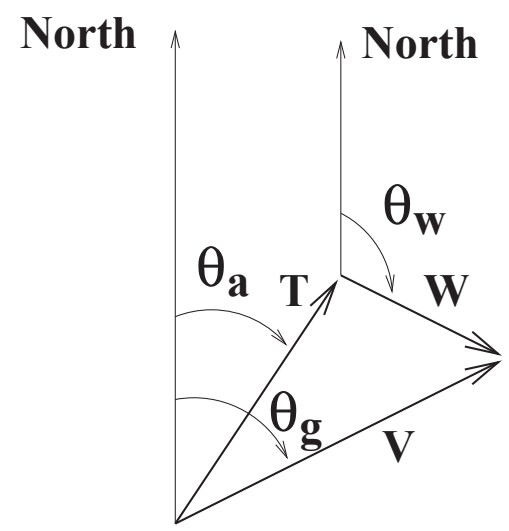

Figure 1. Speed Vector Relations

with $\gamma_{x}=\frac{d v_{x}}{d t}$ and $\gamma_{y}=\frac{d v_{y}}{d t}$. One can show that the air turning rate $\left(\omega_{a}\right)$ and the ground turning rate $\left(\omega_{g}\right)$ are related by the following expression :

$$
\omega_{g}=\left(\frac{T^{2}+T * W * \cos \left(\theta_{a}(t)-\theta_{g}(t)\right)}{T^{2}+W^{2}+2 . T \cdot W \cos \left(\theta_{a}(t)-\theta_{g}(t)\right)}\right) \omega_{a}
$$

Hypothesis We consider en-route traffic (traffic away from airports), with aircraft flying in cruise phase. Aircraft are supposed to fly at constant air speed $\left(T=C^{t e}\right)$ and turn with constant air turning rate $\left(\omega_{a}=C^{t e}\right)$. The average wind is supposed to be constant in the neighborhood of the aircraft.

\section{Observability Conditions}

\section{Mode $S$ Radar}

Like it has been mentioned in the introduction, such radar are able to access on board parameters and especially airspeed vector. Having such measures the system is fully determined at any time as it is shown in the following equations.

$$
\left\{v_{x}=T \sin \left(\theta_{a}\right)+w_{x} \quad v_{y}=T \cos \left(\theta_{a}\right)+w_{y}\right.
$$

In this system there are two equations and two unknowns $\left(w_{x}, w_{y}\right)$; the other parameters are given by the radar $\left(v_{x}, v_{y}, T, \theta_{a}\right)$. A Kalman filter will be used to extract the wind by removing the noise.

\section{Standard Radar}

If measures come from classical radars, only position measures are available $(x, y)$ and two situations have to be taken into account.

$\underline{\text { First situation: One aircraft }}$
In the case where radar measures come from one aircraft, wind may be observed only after two asymmetric turns (meaning three straight lines separated by two turns). As a mater of fact, the first segment brings the following system of equations ;

$$
\left\{v_{1 x}=T \sin \left(\theta_{a 1}\right)+w_{x} \quad v_{1 y}=T \cos \left(\theta_{a 1}\right)+w_{y}\right.
$$

In this system (4), there are 4 unknowns $\left(T, \theta_{a 1}, w_{x}, w_{y}\right)$ and two equations, so two equations are missing.

After the first turn two new equations are added to this system with one extra unknown $\left(\theta_{a 2}\right)$ :

$$
\begin{cases}v_{1 x}=T \sin \left(\theta_{a 1}\right)+w_{x} & v_{1 y}=T \cos \left(\theta_{a 1}\right)+w_{y} \\ v_{2 x}=T \sin \left(\theta_{a 2}\right)+w_{x} & v_{2 y}=T \cos \left(\theta_{a 2}\right)+w_{y}\end{cases}
$$

In this new system (5), there are 5 unknowns $\left(T, \theta_{a 1}, \theta_{a 2}, w_{x}, w_{y}\right)$ and only 4 equations. It is only after the second turn that the system is fully determined (6 unknowns $\left(T, \theta_{a 1}, \theta_{a 2}, \theta_{a 3}, w_{x}, w_{y}\right)$ and 6 equations) :

$$
\begin{cases}v_{1 x}=T \sin \left(\theta_{a 1}\right)+w_{x} & v_{1 y}=T \cos \left(\theta_{a 1}\right)+w_{y} \\ v_{2 x}=T \sin \left(\theta_{a 2}\right)+w_{x} & v_{2 y}=T \cos \left(\theta_{a 2}\right)+w_{y} \\ v_{3 x}=T \sin \left(\theta_{a 3}\right)+w_{x} & v_{3 y}=T \cos \left(\theta_{a 3}\right)+w_{y}\end{cases}
$$

This system has a closed form solution for which the wind is given by :

$$
\begin{aligned}
& w_{x}=\frac{\left(v_{3 y}-v_{2 y}\right) V_{1}^{2}+\left(v_{1 y}-v_{3 y}\right) V_{2}^{2}+\left(v_{2 y}-v_{1 y}\right) V_{3}^{2}}{2\left\{v_{1 y}\left(v_{2 x}-v_{3 x}\right)+v_{2 y}\left(v_{3 x}-v_{1 x}\right)+v_{3 y}\left(v_{1 x}-v_{2 x}\right)\right\}} \\
& w_{y}=\frac{\left(v_{2 x}-v_{3 x}\right) V_{1}^{2}+\left(v_{3 x}-v_{1 x}\right) V_{2}^{2}+\left(v_{1 x}-v_{2 x}\right) V_{3}^{2}}{2\left\{v_{1 y}\left(v_{2 x}-v_{3 x}\right)+v_{2 y}\left(v_{3 x}-v_{1 x}\right)+v_{3 y}\left(v_{1 x}-v_{2 x}\right)\right\}}
\end{aligned}
$$

With

$$
V_{1}=\sqrt{v_{1 x}^{2}+v_{1 y}^{2}} V_{2}=\sqrt{v_{2 x}^{2}+v_{2 y}^{2}} V_{3}=\sqrt{v_{3 x}^{2}+v_{3 y}^{2}}
$$

For both expressions $\left(w_{x}, w_{y}\right)$ the denominators must not be equal to zero meaning that turns have to be asymmetric $\left(\underline{V}_{1} \neq \underline{V}_{2} \neq \underline{V}_{3}\right)$.Having the expressions for $w_{x}$ and $w_{y}$ it is very easy to extract the other unknowns :

$\begin{cases}t_{1 x}=T \sin \left(\theta_{a 1}\right)=v_{1 x}-w_{x} & t_{1 y}=T \cos \left(\theta_{a 1}\right)=v_{1 y}-w_{y} \\ t_{2 x}=T \sin \left(\theta_{a 2}\right)=v_{2 x}-w_{x} & t_{2 y}=T \cos \left(\theta_{a 2}\right)=v_{2 y}-w_{y} \\ t_{3 x}=T \sin \left(\theta_{a 3}\right)=v_{3 x}-w_{x} & t_{3 y}=T \cos \left(\theta_{a 3}\right)=v_{3 y}-w_{y}\end{cases}$

then

$$
\begin{gathered}
T=\sqrt{t_{1 x}^{2}+t_{1 y}^{2}}=\sqrt{t_{2 x}^{2}+t_{2 y}^{2}}=\sqrt{t_{3 x}^{2}+t_{3 y}^{2}} \\
\theta_{a 1}=\arctan \left(\frac{t_{1 x}}{t_{1 y}}\right) \theta_{a 2}=\arctan \left(\frac{t_{2 x}}{t_{2 y}}\right) \theta_{a 3}=\arctan \left(\frac{t_{3 x}}{t_{3 y}}\right)
\end{gathered}
$$


It is also possible to extract a close form for the True Air Speed $(T)$ of the aircraft using only the ground speeds :

$$
T=\frac{\left\|\Delta \vec{v}_{12}\right\| \cdot\left\|\Delta \vec{v}_{13}\right\| \cdot\left\|\Delta \vec{v}_{23}\right\|}{2 .\left|v_{1 y} \cdot\left(v_{2 x}-v_{3 x}\right)+v_{2 y} \cdot v_{3 x}-v_{2 x} \cdot v_{3 y}+v_{1 x} \cdot\left(v_{3 y}-v_{2 y}\right)\right|}
$$

Where $\Delta \vec{v}_{i j}=\vec{v}_{i}-\vec{v}_{j}$ The same kind of close forms can be extracted for the three heading angles $\left(\theta_{a 1}, \theta_{a 2}, \theta_{a 3}\right): \theta_{a i}=\arctan \left(\frac{t_{i x}}{t_{i y}}\right)$ where $\frac{t_{i x}}{t_{i y}}=$

$$
\frac{2 \cdot v_{i x} \cdot A+B}{2 \cdot v_{i y} \cdot A-C}
$$

where

$$
\begin{aligned}
& A=\Delta \vec{v}_{23 x} \cdot v_{1 y}+\Delta \vec{v}_{31 x} \cdot v_{2 y}+\Delta \vec{v}_{12 x} \cdot v_{3 y} \\
& B=\Delta \vec{v}_{23 y} \cdot V_{1}^{2}+\Delta \vec{v}_{31 y} \cdot V_{2}^{2}+\Delta \vec{v}_{12 y} \cdot V_{3}^{2} \\
& C=\Delta \vec{v}_{23 x} \cdot V_{1}^{2}+\Delta \vec{v}_{31 x} \cdot V_{2}^{2}+\Delta \vec{v}_{12 x} \cdot V_{3}^{2}
\end{aligned}
$$

\section{$\underline{\text { Second situation : Two aircraft }}$}

When radar measures are available for two aircraft ( $a$ and $b$ ), only one turn for both trajectories is needed to have enough information for wind estimation. The two first segments bring the following system of equations with 6 unknowns $\left(T_{a}, T_{b}, \theta_{a_{a 1}}, \theta_{a_{b 1}}, w_{x}, w_{y}\right)$ and 4 equations :

$$
\begin{cases}v a_{1 x}=T_{a} \sin \left(\theta_{a_{a 1}}\right)+w_{x} & v a_{1 y}=T_{a} \cos \left(\theta_{a_{a 1}}\right)+w_{y} \\ v b_{1 x}=T_{b} \sin \left(\theta_{a_{b 1}}\right)+w_{x} & v b_{1 y}=T_{b} \cos \left(\theta_{a_{b 1}}\right)+w_{y}\end{cases}
$$

After the second turn, the new systems is fully determined with 8 unknowns $\left(T_{a}, T_{b}, \theta_{a_{a 1}}, \theta_{a_{b 1}}, \theta_{a_{a 2}}, \theta_{a_{b 2}}, w_{x}, w_{y}\right)$ and 8 equations :

$$
\begin{cases}v a_{1 x}=T_{a} \sin \left(\theta_{a_{a 1}}\right)+w_{x} & v a_{1 y}=T_{a} \cos \left(\theta_{a_{a 1}}\right)+w_{y} \\ v b_{1 x}=T_{b} \sin \left(\theta_{a_{b 1}}\right)+w_{x} & v b_{1 y}=T_{b} \cos \left(\theta_{a_{b 1}}\right)+w_{y} \\ v a_{2 x}=T_{a} \sin \left(\theta_{a_{a 2}}\right)+w_{x} & v a_{2 y}=T_{a} \cos \left(\theta_{a_{a 2}}\right)+w_{y} \\ v b_{2 x}=T_{b} \sin \left(\theta_{a_{b 2}}\right)+w_{x} & v b_{2 y}=T_{b} \cos \left(\theta_{a_{b 2}}\right)+w_{y}\end{cases}
$$

The associated closed form of the wind is given by :

$$
\begin{aligned}
& w_{x}=\frac{\left(v b_{1 y}-v b_{2 y}\right)\left(V a_{1}^{2}-V a_{2}^{2}\right)+\left(v a_{2 y}-v a_{1 y}\right)\left(V b_{1}^{2}-V b_{2}^{2}\right)}{2\left\{\left(v a_{1 x}-v a_{2 x}\right)\left(v b_{1 y}-v b_{2 y}\right)-\left(v a_{1 y}-v a_{2 y}\right)\left(v b_{1 x}-v b_{2 x}\right)\right\}} \\
& w_{y}=\frac{\left(v b_{2 x}-v b_{1 x}\right)\left(V a_{1}^{2}-V a_{2}^{2}\right)+\left(v a_{1 x}-v a_{2 x}\right)\left(V b_{1}^{2}-V b_{2}^{2}\right)}{2\left\{\left(v a_{1 x}-v a_{2 x}\right)\left(v b_{1 y}-v b_{2 y}\right)-\left(v a_{1 y}-v a_{2 y}\right)\left(v b_{1 x}-v b_{2 x}\right)\right\}}
\end{aligned}
$$

with

$$
\begin{array}{ll}
V a_{1}=\sqrt{v a_{1 x}^{2}+v a_{1 y}^{2}} & V a_{2}=\sqrt{v a_{2 x}^{2}+v a_{2 y}^{2}} \\
V b_{1}=\sqrt{v b_{1 x}^{2}+v b_{1 y}^{2}} & V b_{2}=\sqrt{v b_{2 x}^{2}+v b_{2 y}^{2}}
\end{array}
$$

and $\left(\underline{V} a_{1} \neq \underline{V} a_{2} ; \underline{V} b_{1} \neq \underline{V} b_{2}\right)$.

Like for the one aircraft case, it is very easy to extract the other unknowns :

$$
\begin{cases}t a_{1 x}=v a_{1 x}-w_{x} & t a_{1 y}=v a_{1 y}-w_{y} \\ t b_{1 x}=v b_{1 x}-w_{x} & t b_{1 y}=v b_{1 y}-w_{y} \\ t a_{2 x}=v a_{2 x}-w_{x} & t a_{2 y}=v a_{2 y}-w_{y} \\ t b_{2 x}=v b_{2 x}-w_{x} & t b_{2 y}=v b_{2 y}-w_{y}\end{cases}
$$

then

$$
\left\{\begin{array}{l}
T_{a}=\sqrt{t a_{1 x}^{2}+t a_{1 y}^{2}}=\sqrt{t a_{2 x}^{2}+t a_{2 y}^{2}} \\
T_{b}=\sqrt{t b_{1 x}^{2}+t b_{1 y}^{2}}=\sqrt{t b_{2 x}^{2}+t b_{2 y}^{2}}
\end{array}\right.
$$

A close form of $T_{a}$ and $T_{b}$ can be extracted :

$$
\begin{aligned}
& T_{a}=\sqrt{t a_{1 x}^{2}+t a_{1 y}^{2}}=\sqrt{t a_{2 x}^{2}+t a_{2 y}^{2}} \\
& t a_{1 x}^{2}=\frac{\left[\Delta v b_{12 y}\left(V a_{1}^{2}-2 . \Delta v a_{12 x} \cdot v a_{1 x}-V a_{2}^{2}\right)+\Delta v a_{12 y}\left(2 . \Delta v b_{12 x} \cdot v a_{1 x}-V b_{1}^{2}+V b_{2}^{2}\right)\right]^{2}}{4\left(\Delta v a_{12 y} \cdot \Delta v b_{12 x}-\Delta v a_{12 x} \cdot \Delta v b_{12 y}\right)^{2}} \\
& t a_{1 y}^{2}=\frac{\left[\Delta v b_{12 x}\left(V a_{1}^{2}-2 . \Delta v a_{12 y} \cdot v a_{1 y}-V a_{2}^{2}\right)+\Delta v a_{12 x}\left(2 . \Delta v b_{12 y} \cdot v a_{1 y}-V b_{1}^{2}+V b_{2}^{2}\right)\right]^{2}}{4\left(\Delta v a_{12 y} \cdot \Delta v b_{12 x}-\Delta v a_{12 x} \cdot \Delta v b_{12 y}\right)^{2}} \\
& T_{b}=\sqrt{t b_{1 x}^{2}+t b_{1 y}^{2}}=\sqrt{t b_{2 x}^{2}+t b_{2 y}^{2}} \\
& t b_{1 x}^{2}=\frac{\left[\Delta v b_{12 y}\left(V a_{1}^{2}-2 \cdot \Delta v a_{12 x} \cdot v b_{1 x}-V a_{2}^{2}\right)+\Delta v a_{12 y}\left(2 \cdot \Delta v b_{12 x} \cdot v b_{1 x}-V b_{1}^{2}+V b_{2}^{2}\right)\right]^{2}}{4\left(\Delta v a_{12 y} \cdot \Delta v b_{12 x}-\Delta v a_{12 x} \cdot \Delta v b_{12 y}\right)^{2}} \\
& t b_{1 y}^{2}=\frac{\left[\Delta v b_{12 x}\left(V a_{1}^{2}-2 . \Delta v a_{12 y} \cdot v b_{1 y}-V a_{2}^{2}\right)+\Delta v a_{12 x}\left(2 . \Delta v b_{12 y} \cdot v b_{1 y}-V b_{1}^{2}+V b_{2}^{2}\right)\right]^{2}}{4\left(\Delta v a_{12 y} \cdot \Delta v b_{12 x}-\Delta v a_{12 x} \cdot \Delta v b_{12 y}\right)^{2}} \\
& \begin{cases}\theta_{a_{a 1}}=\arctan \left(\frac{t a_{1 x}}{t a_{1 y}}\right) & \theta_{a_{a 2}}=\arctan \left(\frac{t a_{2 x}}{t a_{2 y}}\right) \\
\theta_{a_{b 1}}=\arctan \left(\frac{t b_{1 x}}{t b_{1 y}}\right) & \theta_{a_{b 2}}=\arctan \left(\frac{t b_{2 x}}{t b_{2 y}}\right)\end{cases}
\end{aligned}
$$

Based on those observability conditions, several wind estimation models have been developed for which some of them use a Kalman filter. This filter is now described in the following section.

\section{Kalman Filtering}

\section{Linear Form}

The Kalman filter is named after Rudolph E.Kalman, who in 1960 published his famous paper describing a recursive solution to the discrete-data linear filtering problem [12], [10], [18]. The Kalman filter is essentially a set of mathematical equations that implements a predictor-corrector type estimator that is 
optimal in the sense that it minimizes the estimated error covariance (when some presumed conditions are met). It has been successfully applied in many real problems [14], [5], [3], [2], [1]. The following description of the Kalman filter is mainly due to G.Welch and G.Bishop [18].

The Kalman filter addresses the general problem of trying to estimate the state $\underline{X}(k) \in \mathcal{R}^{n}$ of a discrete controlled process that is governed by the linear stochastic difference equation call evolution equation :

$$
\underline{X}(k+1)=\underline{F}(k) \cdot \underline{X}(k)+\underline{G}(k) \cdot \underline{\hat{U}}(k)+\underline{v}(k)
$$

with a measurement $\underline{Z}(k) \in \mathcal{R}^{m}$ :

$$
\underline{Z}(k)=\underline{H}(k) \cdot \underline{X}(k)+\underline{w}(k)
$$

The $n . n$ matrix $\underline{F}(k)$ in the evolution equation (14) relates the state at the present step $k$ to the state at the next step $k+1$, in the absence of either a driving function or process noise. The n.p matrix $\underline{G}(k)$ relates the optional control input $\underline{\underline{U}}(k) \in \mathcal{R}^{p}$ to the state $\underline{X}$. The $n . m$ matrix $\underline{H}(k)$ in the measurement equation (15) relates the state to the measurement $\underline{Z}(k)$. The random variables $\underline{v}(k)$ and $\underline{w}(k)$ represent the process and measurement noise (respectively). They are assumed to be independent (of each other), centered, white, and with normal probability distributions. Furthermore the optional control is disturbed by a random variable $\underline{n}(k)$ $(\underline{\hat{U}}(k)=\underline{U}(k)+\underline{n}(k))$ which will be supposed to have a normal probability distribution also.

$$
\left\{\begin{array}{lll}
p(\underline{v}(k)) & \sim \mathcal{N}(0, \underline{Q}(k)) \\
p(\underline{w}(k)) & \backsim \mathcal{N}(0, \underline{R}(k)) \\
p(\underline{n}(k)) & \sim \mathcal{N}(0, \underline{N}(k))
\end{array}\right.
$$

So we have :

$$
\begin{array}{ll}
E[\underline{v}(k)]=0 & E\left[\underline{v}(k) \underline{v}(j)^{T}\right]=Q(k) \cdot \delta_{k j} \\
E[\underline{w}(k)]=0 & E\left[\underline{w}(k) \underline{w}(j)^{T}\right]=\underline{R}(k) \cdot \delta_{k j} \\
E[\underline{n}(k)]=0 & E\left[\underline{n}(k) \underline{n}(j)^{T}\right]=\underline{N}(k) \cdot \delta_{k j}
\end{array}
$$

where ${ }^{T}$ is the transpose operator and $\delta_{k j}$ is the Kronecker symbol which is 1 if $k=j$ and 0 if $k \neq j$.

We define $\underline{\hat{X}}(k+1 / k) \in \mathcal{R}^{n}$ to be the a priori state estimate at step $k+1$ given knowledge of the process prior to step $k+1$, and $\underline{\hat{X}}(k+1 / k+1) \in \mathcal{R}^{n}$ to be the a posteriori state estimate at step $k+1$ given measurement $\underline{Z}(k+1)$. We can then define a priori and a posteriori estimate error as :

$$
\left\{\begin{array}{l}
\underline{e}(k+1 / k)=\underline{X}(k+1)-\underline{\hat{X}}(k+1 / k) \\
\underline{e}(k+1 / k+1)=\underline{X}(k+1)-\underline{\hat{X}}(k+1 / k+1)
\end{array}\right.
$$

The a priori estimate error covariance is then :

$$
\underline{P}(k+1 / k)=E\left[\underline{e}(k+1 / k) \underline{e}(k+1 / k)^{T}\right]
$$

and the a posteriori estimate error covariance is :

$$
\begin{gathered}
\underline{P}(k+1 / k+1)= \\
E\left[\underline{e}(k+1 / k+1) \underline{e}(k+1 / k+1)^{T}\right]
\end{gathered}
$$

In deriving the equation for the Kalman filter, we begin with the goal of finding an equation that computes an a posteriori state estimate $\underline{\hat{X}}(k+1 / k+1)$ as a linear combination of an a priori estimate $\underline{\hat{X}}(k+1 / k)$ and a weighted difference between an actual measurement $\underline{Z}(k+1)$ and a measurement prediction $\underline{H} \cdot \underline{\hat{X}}(k+1 / k)$ :

$$
\begin{gathered}
\hat{X}(k+1 / k+1)=\underline{\hat{X}}(k+1 / k)+ \\
\underline{K}(k+1) \cdot(\underline{Z}(k+1)-\underline{H} \cdot \underline{\hat{X}}(k+1 / k))
\end{gathered}
$$

The difference $(\underline{Z}(k+1)-\underline{H} \cdot \underline{\hat{X}}(k+1 / k))$ in equation 21 is called the measurement innovation, or the residual. The residual reflects the discrepancy between the predicted measurement $\underline{H} \cdot \underline{\hat{X}}(k+1 / k)$ and the actual measurement $\underline{Z}(k+1)$. A residual of zero means that the two are in complete agreement.

The $n . m$ matrix $\underline{K}(k+1)$ in equation 21 is chosen to be the gain or blending factor that minimizes the a posteriori error covariance 20 . This minimization can be accomplished by first substituting equation 21 into the above definition for $\underline{e}(k+1 / k+1)$, substituting that into equation 20, performing the indicated expectations, taking the derivative of the trace of the result with respect to $\underline{K}(k+1)$, setting that result equal to zero, and then solving for $\underline{K}(k+1)$. One form of the resulting $\underline{K}(k+1)$ that minimizes equation 20 is given by :

$$
\begin{gathered}
\underline{K}(k+1)=\underline{P}(k+1 / k) \cdot \underline{H}(k+1)^{T} . \\
{\left[\underline{H}(k+1) \underline{P}(k+1 / k) \underline{H}(k+1)^{T}+\underline{R}(k+1)\right]^{-1}}
\end{gathered}
$$

Looking at equation 22 we see that as the measurement error covariance $\underline{R}(k+1)$ approaches zero, the gain $\underline{K}(k+1)$ weights the residual more heavily. Specifically :

$$
\lim _{\underline{R}(k+1) \rightarrow \underline{\underline{K}}} \underline{K}(k+1)=\underline{H}^{-1}
$$

On the other hand, as the a priori estimate error covariance $\underline{P}(k+1 / k)$ approaches zero, the gain $\underline{K}(k+1)$ weights the residual less heavily. Specifically:

$$
\lim _{\underline{P}(k+1 / k) \rightarrow \underline{0}} \underline{K}(k+1)=0
$$


As the measurement error covariance $\underline{R}(k+1)$, the actual measurement $\underline{Z}(k+1)$ is "trusted" more and more, while the predicted measurement $\underline{H} \cdot \underline{\hat{X}}(k+1 / k)$ is trusted less and less. On the other hand, as the a priori estimate error covariance $\underline{P}(k+1 / k)$ approaches zero the actual measurement $\underline{Z}(k+1)$ is trusted less and less, while the predicted measurement $\underline{H} \cdot \underline{\hat{X}}(k+$ $1 / k)$ is trusted more and more.

The Kalman filter estimates a process by using a form of feedback control : the filter estimates the process state at some time and then obtains feedback in the form of (noisy) measurements. As such, the equation for the Kalman filter fall into two groups : time update equations and measurement update equations. The time update equations are responsible for projecting forward (in time) the current state and error covariance estimates to obtain the a priori estimates for the next time step. The measurement update equations are responsible for the feedback -i.e. for incorporating a new measurement into the a priori estimate to obtain an improved estimate.

The time update equations are the following :

$$
\left\{\begin{aligned}
\hat{X}(k+1 / k)= & \underline{F}(k) \cdot \underline{X}(k)+\underline{G}(k) \cdot \underline{U}(k) \\
\underline{P}(k+1 / k)= & \underline{F}(k) \cdot \underline{P}(k / k) \cdot \underline{F}(k)^{T}+ \\
& \underline{G}(k) \cdot \underline{N}(k) \cdot \underline{G}(k)^{T}+\underline{Q}(k)
\end{aligned}\right.
$$

The first equation is the state prediction and the second one gives the associated covariance. The measurement update equations are the following:

$$
\left\{\begin{array}{l}
\underline{K}(k+1)=\underline{P}(k+1 / k) \cdot \underline{H}(k+1)^{T} . \\
{\left[\underline{H}(k+1) \underline{P}(k+1 / k) \underline{H}(k+1)^{T}+\underline{R}(k+1)\right]^{-1}} \\
\underline{\hat{X}}(k+1 / k+1)=\underline{\hat{X}}(k+1 / k)+\underline{K}(k+1) . \\
{[\underline{Z}(k+1)-\underline{H}(k+1) \cdot \underline{\hat{X}}(k+1 / k)]} \\
\underline{P}(k+1 / k+1)=[\underline{\underline{K}}(k+1) \cdot \underline{H}(k+1)] . \\
\underline{P}(k+1 / k)
\end{array}\right.
$$

The first task during the measurement update is to compute the Kalman gain, $\underline{K}(k+1)$. The next step is to actually measure the process to obtain $\underline{Z}(k+1)$, and then to generate an a posteriori state estimated by incorporating the measurement. The final step is to obtain an a posteriori error covariance estimate.

After each time and measurement update pair, the process is repeated with the previous a posteriori estimates used to project or predict the new a priori estimate. In order to initiate the process, the first initial state and the associated covariance has to be set up. Usually, the first state is build with an initial measure and the associated covariance is set up with the measure covariance or with a big values, because the filter has no confidence in its initial state (it comes from a measure). This Kalman filter has been used in our wind estimation problem for which the associated models are now presented.

\section{Extended Form}

The standard Kalman filter addresses the general problem of trying to estimate the state $\underline{X}(k) \in \mathcal{R}^{n}$ of a discrete-time controlled process that is governed by a linear stochastic difference equation. But what happens if the process to be estimated and (or) the measurement relationship to the process is non-linear? A Kalman filter that linearized about the current mean and covariance is referred to as an extended Kalman filter or EKF. We can linearize the estimation around the current estimate using the partial derivative of the process and measurement functions to compute estimates even in the face of non-linear relationships. Let us assume that our process again has a state vector $\underline{X}(k) \in \mathcal{R}^{n}$, but that process is now governed by the non-linear stochastic difference equation :

$$
\underline{X}(k+1)=\underline{\mathcal{F}}[k, \underline{X}(k), \underline{U}(k)]+\underline{v}(k)
$$

with a measurement $\underline{Z}(k) \in \mathcal{R}^{m}$ that is :

$$
\underline{Z}(k+1)=\underline{\mathcal{H}}[k, \underline{X}(k)]+\underline{w}(k)
$$

where the random variable $\underline{v}(k)$ and $\underline{w}(k)$ again represent the process and measurement noise. The new time update equations are now the followings :

$$
\left\{\begin{array}{l}
\hat{X}(k+1 / k)=\frac{\mathcal{F}}{\mathcal{P}}[k, \underline{X}(k), \underline{U}(k)] \\
\underline{P}(k+1 / k)=\frac{\underline{\mathcal{F}}_{X}(k) \cdot \underline{P}(k / k) \cdot \underline{\mathcal{F}}_{X}(k)^{T}+}{\underline{\mathcal{F}}_{\underline{U}}(k) \cdot \underline{N}(k) \cdot \underline{\mathcal{F}}_{\underline{X}}(k)^{T}+\underline{Q}(k)}
\end{array}\right.
$$

where $\underline{\mathcal{F}}_{X}(k)$ is the Jacobian matrix of partial derivatives of $\underline{\mathcal{F}}$ with respect to the vector $\underline{X}$ and is given by the following :

$$
\underline{\mathcal{F}}_{\underline{X}}(k) \hat{=}\left[\nabla \underline{X}\left(\underline{\mathcal{F}}[k, \underline{X}, \underline{U}]^{T}\right)\right]_{\underline{X}=\underline{\hat{X}}(k / k), \underline{U}=\underline{\hat{U}}(k)}^{T}
$$

where

$$
\nabla \underline{\underline{ }} \hat{=}\left[\frac{\partial}{\partial_{S_{1}}}, \frac{\partial}{\partial_{S_{2}}}, \ldots, \frac{\partial}{\partial_{S_{n} s}}\right]
$$

is the differential operator. 
The linearized measurement update is now given by :

$$
\left\{\begin{array}{l}
\underline{K}(k+1)=\underline{P}(k+1 / k) \cdot \underline{\mathcal{H}}_{X}(k+1)^{T} . \\
{\left[\underline{\mathcal{H}}(k+1) \cdot \underline{P}(k+1 / k) \underline{\mathcal{H}}_{X}(k+1)^{T}+\underline{R}(k+1)\right]^{-1}} \\
\underline{\hat{X}}(k+1 / k+1)=\underline{X}(k+1 / k)+\underline{K}(k+1) . \\
{[\underline{Z}(k+1)-\underline{\mathcal{H}}(k, \underline{\hat{X}}(k+1 / k))]} \\
\underline{P}(k+1 / k+1)=\left[\underline{I}-\underline{K}(k+1) \cdot \underline{\mathcal{H}}_{\underline{X}}(k+1)\right] . \\
\underline{P}(k+1 / k)
\end{array}\right.
$$

Unlike its linear counterpart, the extended Kalman filter is not an optimal estimator. In addition, if the initial estimate of the state is wrong, or if the process is modeled incorrectly, the filter may quickly diverge, owing to its linearization. Another problem with the extended Kalman filter is that the estimated covariance matrix tends to underestimate the true covariance matrix and therefore risks becoming inconsistent in the statistical sense without the addition of "stabilizing noise". When the state transition and observation models are highly non-linear, the extended Kalman filter can give particularly poor performance [11].This is because only the mean is propagated through the non-linearity.

The Unscented Kalman Filter(UKF) is another non linear extension of the linear Kalman filter for which both mean vector and covariance matrix are propagated through the non-linearity. A detailed description of the UKF is given in appendix[A].

Having described our problem and some estimation techniques, the next section introduces the models which haves been used for our experiments.

\section{Models}

\section{Model with Air Speed and Air Turning Rate (Model 1)}

The state vector is given by :

$$
\underline{X}(k)=\left[x(k) y(k) t_{x}(k) t_{y}(k) w_{x}(k) w_{y}(k)\right]^{T}
$$

where $x(k), y(k)$ is the position, $t_{x}(k), t_{y}(k)$ the True Air Speed (TAS) and $w_{x}(k), w_{y}(k)$ the wind. The measure vector consists in the radar position and the true air speed :

$$
\underline{Z}(k)=\left[x_{m}(k) y_{m}(k) t_{x_{m}}(k) t_{y_{m}}(k)\right]^{T}
$$

Having access to the air turning rate $\left(\omega_{a}\right)$, it can be included in the prediction matrix. The structure of the system is the following :

$$
\underline{X}(k+1)=\underline{F}(k) \cdot \underline{X}(k)+\underline{v}(k)
$$

where

$$
\begin{aligned}
& \underline{F}(k)=\left[\begin{array}{cccccc}
1 & 0 & C_{1}\left(\omega_{a}\right) & C_{2}\left(\omega_{a}\right) & \Delta_{t} & 0 \\
0 & 1 & -C_{2}\left(\omega_{a}\right) & C_{1}\left(\omega_{a}\right) & 0 & \Delta_{t} \\
0 & 0 & C_{3}\left(\omega_{a}\right) & C_{4}\left(\omega_{a}\right) & 0 & 0 \\
0 & 0 & -C_{4}\left(\omega_{a}\right) & C_{3}\left(\omega_{a}\right) & 0 & 0 \\
0 & 0 & 0 & 0 & 1 & 0 \\
0 & 0 & 0 & 0 & 0 & 1
\end{array}\right] \\
& \underline{Z}(k)=\left[\begin{array}{cccccc}
1 & 0 & 0 & 0 & 0 & 0 \\
0 & 1 & 0 & 0 & 0 & 0 \\
0 & 0 & 1 & 0 & 0 & 0 \\
0 & 0 & 0 & 1 & 0 & 0
\end{array}\right] . \underline{X}(k)+\underline{w}(k)
\end{aligned}
$$

where

$$
\left\{\begin{array}{l}
C_{1}\left(\omega_{a}(k)\right)=\frac{\sin \left(\omega_{a}(k) \Delta_{t}\right)}{\omega_{a}(k)} \\
C_{2}\left(\omega_{a}(k)\right)=\frac{1-\cos \left(\omega_{a}(k) \Delta_{t}\right)}{\omega_{a}(k)} \\
C_{3}\left(\omega_{a}(k)\right)=\cos \left(\omega_{a}(k) \Delta_{t}\right) \\
C_{4}\left(\omega_{a}(k)\right)=\sin \left(\omega_{a}(k) \Delta_{t}\right)
\end{array}\right.
$$

The model being exact, the model noise covariance matrix $\underline{R}=\underline{0}$. The measure noise covariance matrix $\underline{R}$ is given by :

$$
\underline{R}=\left[\begin{array}{cccc}
\sigma_{p}^{2} & 0 & 0 & 0 \\
0 & \sigma_{p}^{2} & 0 & 0 \\
0 & 0 & \sigma_{T}^{2} & 0 \\
0 & 0 & 0 & \sigma_{T}^{2}
\end{array}\right]
$$

with $\sigma_{p}=100$ meters and $\sigma_{T}=0.2$ kts.

\section{Model with Air Speed only (model 2)}

The state vector is given by :

$$
\underline{X}(k)=\left[x(k) y(k) t_{x}(k) t_{y}(k) w_{x}(k) w_{y}(k)\right]^{T}
$$

The measure vector consists in the radar position and the true air speed :

$$
\underline{Z}(k)=\left[x_{m}(k) y_{m}(k) t_{x_{m}}(k) t_{y_{m}}(k)\right]^{T}
$$

The prediction matrix is now given by :

$$
\underline{F}(k)=\left[\begin{array}{cccccc}
1 & 0 & \Delta_{t} & 0 & \Delta_{t} & 0 \\
0 & 1 & 0 & \Delta_{t} & 0 & \Delta_{t} \\
0 & 0 & 1 & 0 & 0 & 0 \\
0 & 0 & 0 & 1 & 0 & 0 \\
0 & 0 & 0 & 0 & 1 & 0 \\
0 & 0 & 0 & 0 & 0 & 1
\end{array}\right]
$$


The measure matrix $\underline{H}$ and the associated covariance matrix $\underline{R}$ are the same as in the first model. This model is linear but is false for the air speed vector evolution. In order to take into account this model error, the following covariance matrix $(Q)$ is included in the filter : $\underline{Q}$ is given by :

$$
\underline{Q}=\left[\begin{array}{cccc}
0 & 0 & 0 & 0 \\
0 & 0 & 0 & 0 \\
0 & 0 & 1.0 & 0 \\
0 & 0 & 0 & 1.0
\end{array}\right]
$$

\section{Model with Turning Rate Only (Model 3)}

This model is the same as the first one with the following measure vector ;

$$
\underline{Z}(k)=\left[x_{m}(k) y_{m}(k)\right]^{T}
$$

and the associated measure equation

$$
\underline{Z}(k)=\left[\begin{array}{cccccc}
1 & 0 & 0 & 0 & 0 & 0 \\
0 & 1 & 0 & 0 & 0 & 0
\end{array}\right] \cdot \underline{X}(k)+\underline{w}(k)
$$

\section{Models Without Command}

\section{Model using Kalman Filter (model 4)}

Having no access to the turning rate, this parameter may be extracted by a Kalman filter $\left(\omega_{a}\right.$ included in the state vector). If the turning rate is included in the state vector, the evolution of the system is not linear and an EKF or an UKF has to be used to manage such state vector.

Model using turns (model 5)

As it has been shown in the previous section (observability conditions), wind may be extracted by observing ground radar track during turns.

The key element of this approach is the turning rate detector based on equation (1). When $\omega_{g}$ is greater than a given threshold, the aircraft is consider to be in turn. Based on this turn detector, straight line segment are easily identified for which ground speed vector averages are computed. Speed vector estimates in straight lines are given by the framework of Figure 2. In this figure, the turns are first detected and a counter is then used to select the right averaging process in order to compute ground speed estimates. When two aircraft are used for wind estimation, the same framework is duplicated and counters select only two averaging blocks in order to produce the following estimates $: \underline{V}_{a 1}, \underline{V}_{a 2}, \underline{V}_{b 1}, \underline{V}_{b 2}$.

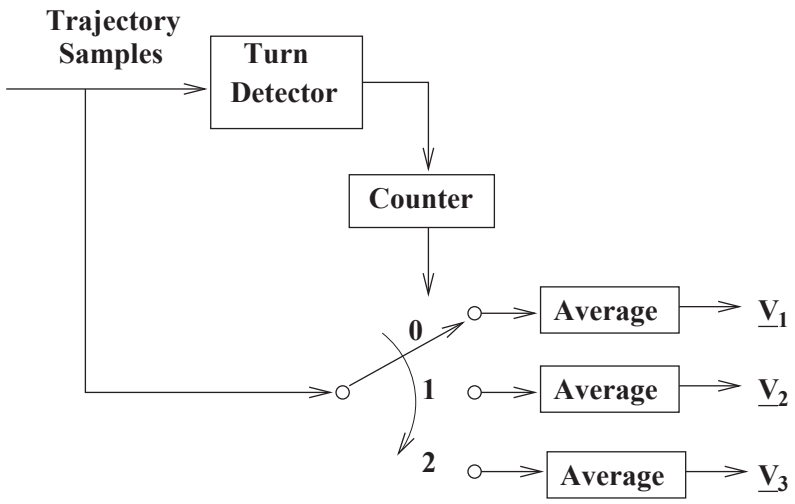

Figure 2. Test Framework

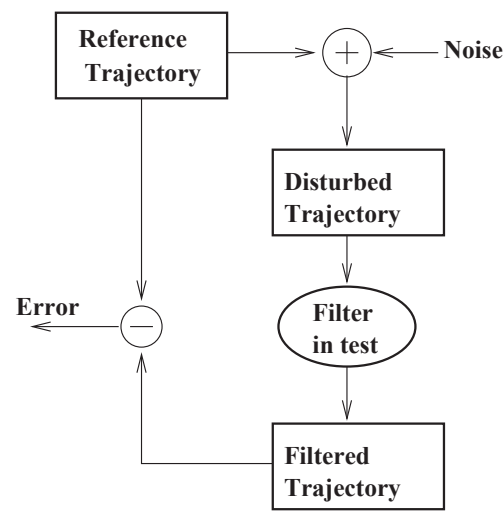

Figure 3. Test Framework

\section{Results}

\section{Simulation Framework}

In order to test and compare our models, a radar tracker simulator has been used to produce the reference trajectory which has been disturbed with a Gaussian noise. The disturbed trajectory is then filtered by a Kalman filters which generates the estimated trajectory which is then compared to the reference trajectory. This framework is summarized on Figure 3. For all experiments, a wind of $40 \mathrm{kts}$ has been used with $\theta_{w}=240^{\circ} \Rightarrow \frac{W}{T}=[-34.64 k t s,-20 k t s]^{T}=$ $[-17.82 \mathrm{~m} / \mathrm{s},-10.28 \mathrm{~m} / \mathrm{s}]^{T}$.

\section{Models with Mode-S radar data (model 1,2 and 3)}

The first trajectory used for our experiments is built with 3 straight lines (20 minutes for each) connected with turns as it can be seen on figure 4. This trajectory has been disturbed by a Gaussian noises for which the means are zero and the standard deviations are the 


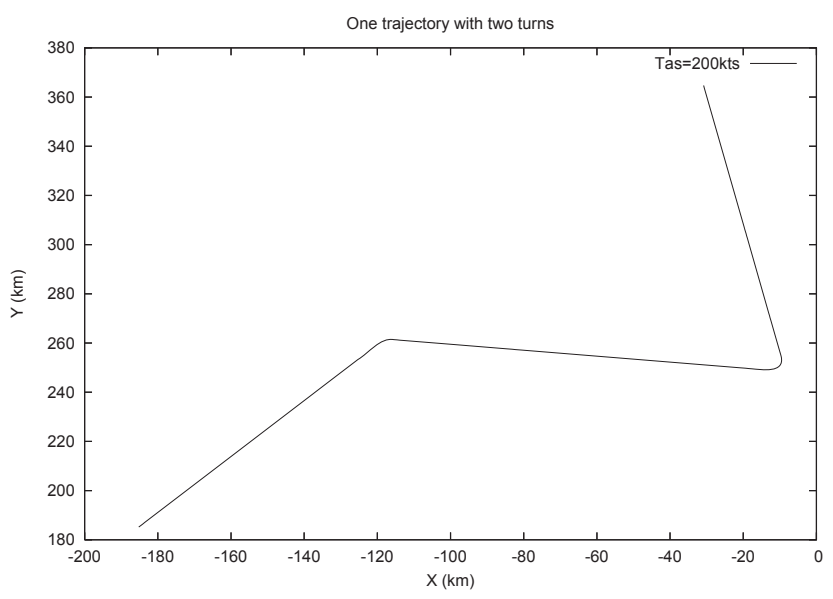

Figure 4. First Trajectory. Each straight lines last 20 minutes. The turning rate of the first turn is $1 \mathrm{deg}$ and $-1 \mathrm{deg}$ for the second turn. The aircraft is considered to fly on cruise phase (vertical speed equal zero).

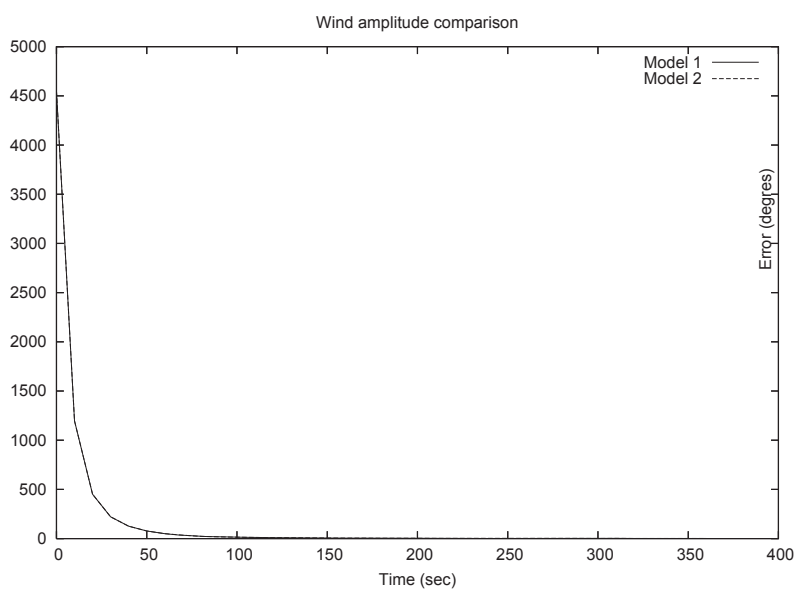

Figure 5. Wind Strength ConvergencePhase for the Models 1 and 2

following $: \sigma_{\text {pos ition }}=100 \mathrm{~m}$ and $\sigma_{T A S}=0.2 \mathrm{kts}$. Those values are given by the performance of the actual radar trackers.

Results for models 1 and 2

Models 1 and 2 have been tried on this trajectory for which the residual errors on the wind estimates are given on figure $5,6,7$ and 8 . The figures 5 and 6 show the residual error for the wind strength estimate. Figure 5 shows the convergence phase of the filter (first 6 minutes) and figure 6 shows the residual error after the convergence phase. The same kind of results are given on figures 7 and 8 for the residual wind angle estimates. The models 1 and 2 have the same evolution during the convergence phase which

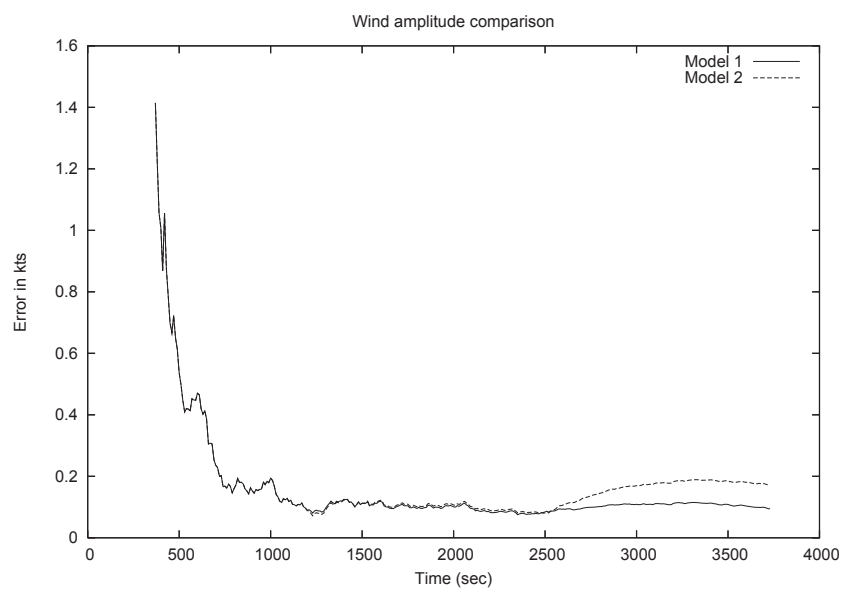

Figure 6. Residual Wind Strength error for Model 1 and 2

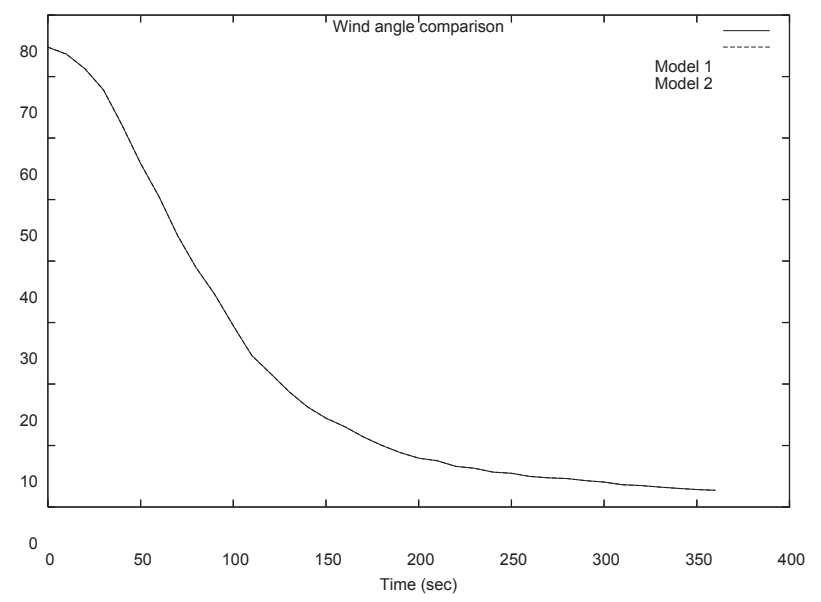

Figure 7. Wind Angle Convergence Phase for the Model 1 and 2

lasts about 2 minutes. After this convergence phase, the wind strength estimate error stays below $0.2 \mathrm{kts}$ for both models 6 . The model 1 has a more accurate behavior at the end of the simulation with an error which stay below $0.1 \mathrm{kts}$. The convergence phase for the wind angle estimate last longer than for the wind strength (about 6 minutes (see figure 7)). After this convergence phase the wind angle residual error stays below 1 degree (in absolute value) for both model. As for the wind strength estimation, the second model (with turning rate) produce a better estimate. The first model is disturbed by the turns.

Both models model are able to produce accurate wind estimate all along the trajectory (after the convergence phase) even during the turns.

Results for models 3 


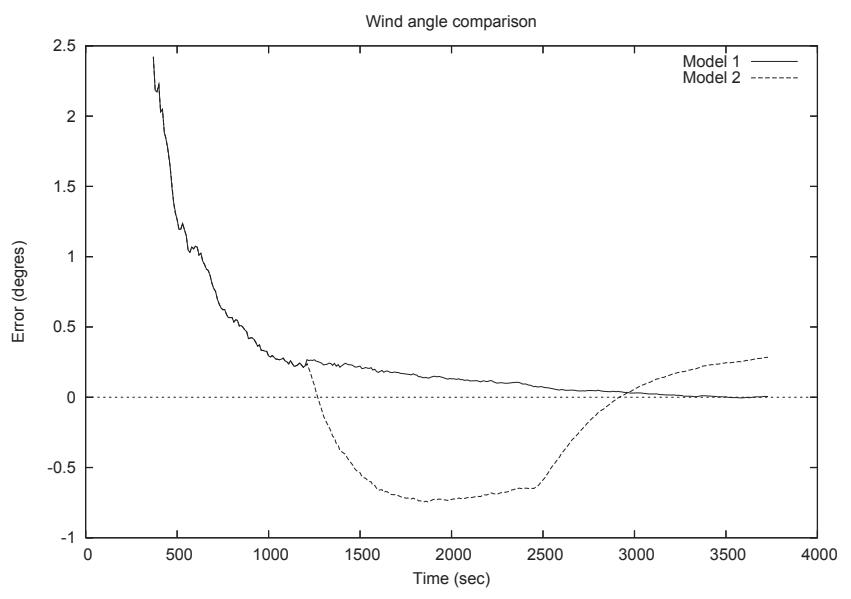

Figure 8. Residual Wind Angle Error for Model 1 and 2

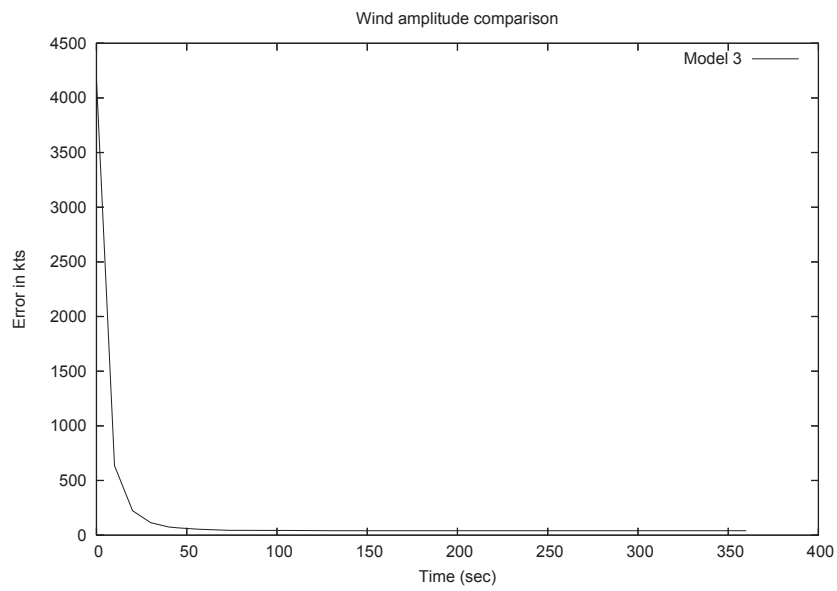

Figure 9.Convergence Phase of the Wind Strength error for Model 2

is not able to converge and model 3 (which is models 2 without air speed measure) has to wait the second turn to be able to produce an estimate of the wind (see figure $9,10,11$ and 12). During the convergence phase (figure 9), model 3 reduces the wind strength error till 40kts which is the limit for such estimation because the Kalman filter is not able to distinguish in the position measures the part coming from the air speed and the part coming from the wind. After the convergence phase (see figure 10) the filter has to wait the second turn in order to be able to produce a reliable estimate of the wind strength (as it has been previously shown (observability conditions), it is only after the second turn that the wind observability conditions are met). As for the wind strength error, the wind angle error reach zero after the second turn.

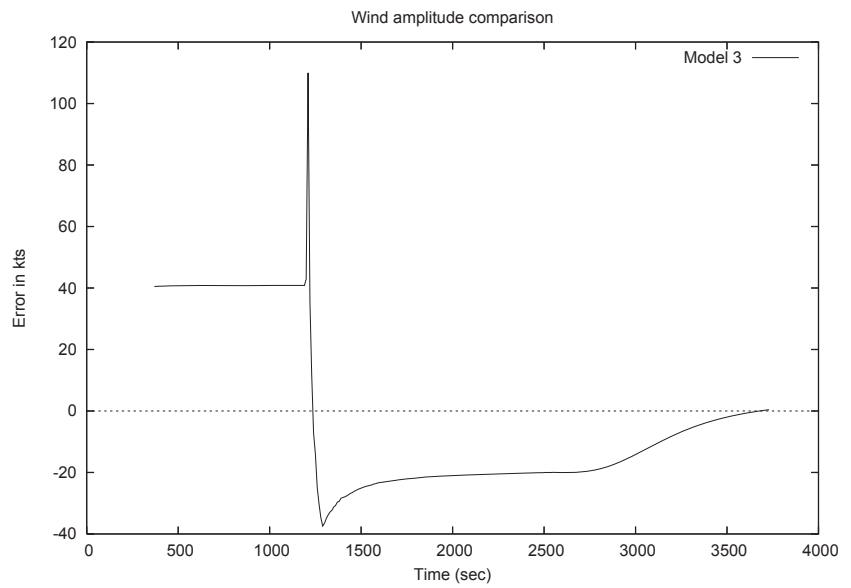

Figure 10. Residual Wind Strength Error for Model 3

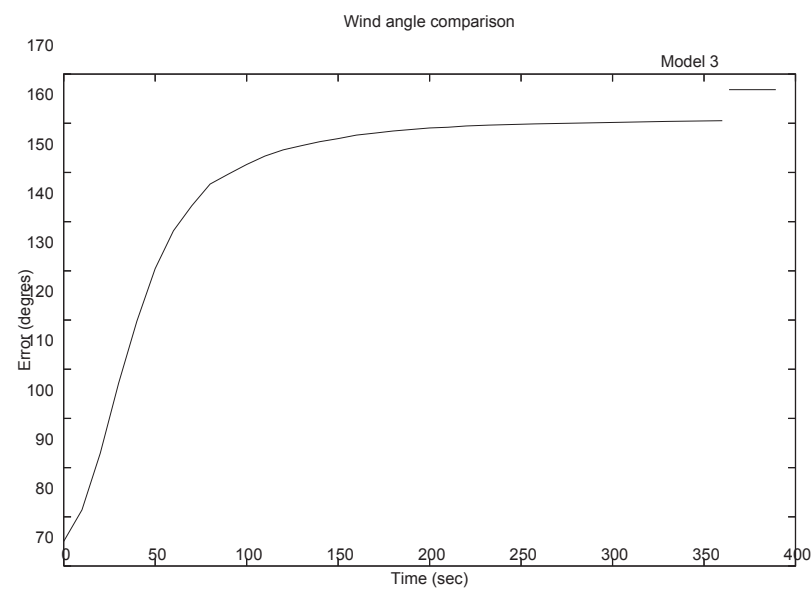

Figure 11. Convergence Phase of theWind Angle Error for Model 3

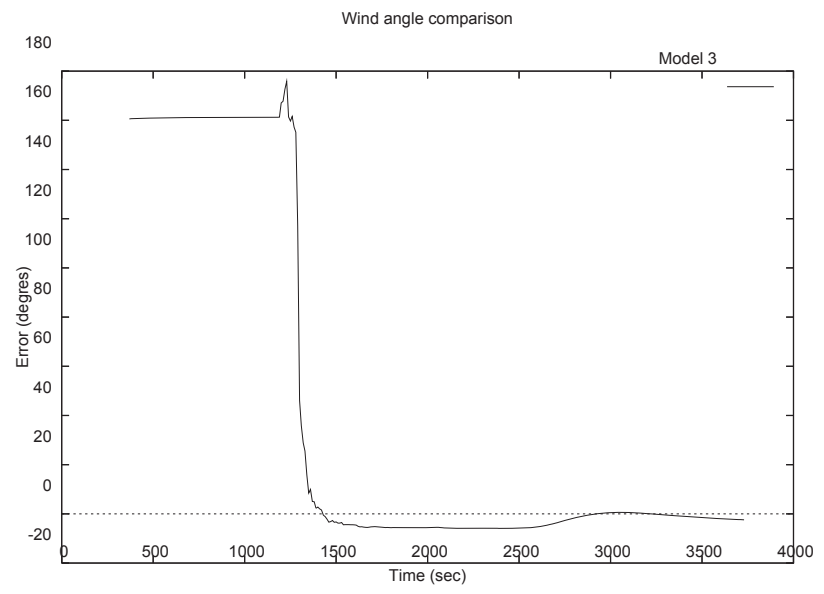

Figure 12. Residual Wind Angle Error for Model 3 


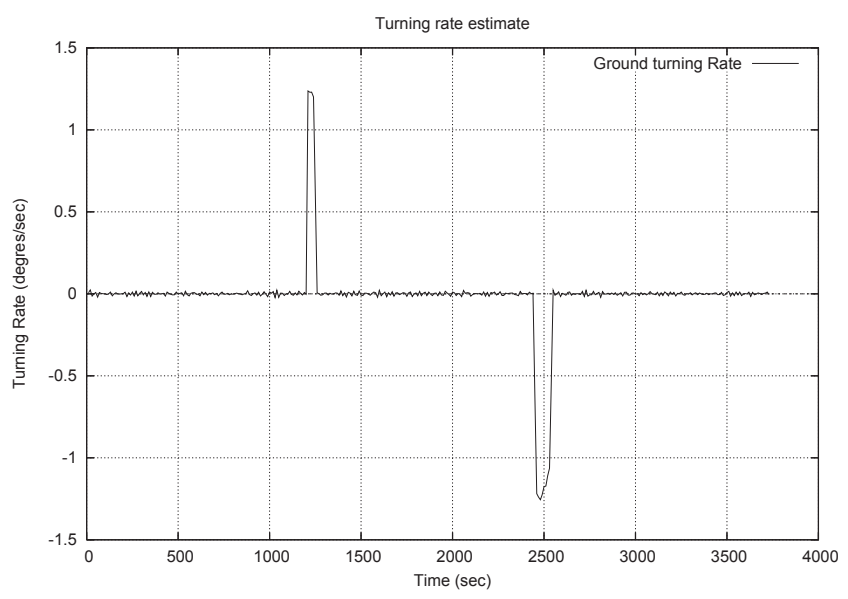

Figure 13. Ground Turning Rate Estimate for the First Trajectory

During the convergence phase the wind angle error reach $160 \mathrm{deg}$ (see figures 11 and 12).

\section{Models with Classical Radar DataModel 4 and 5)}

\section{Results for models 4}

When on board measures (TAS, heading, turning rate) are not available, the turning rate has to be included in the state vector inducing a non linear prediction equation of the filter. In order to address such non linear evolution, an EKF and an UKF has been developed and tested on the first trajectory. Both filters are not able to estimate the wind vector even after the second turn. This behavior is due to the residual error of the Taylor expansion used in the EKF or in the UKF. Based on the observation (positions) the filter is not able to find the part due to the wind and the one due to the error coming from the Taylor expansion.

\section{$\underline{\text { Results for model } 5}$}

Based on the turning rate estimate given by equation 1 , it is very easy to build a turn detector when $\left|\omega_{g}\right|>$ threshold. The ground vector speed has been disturbed by a Gaussian noise with zero mean and $0.2 \mathrm{kts}$ standard deviation (performance of the actual trackers). This method has been used on the first trajectory. The ground turning rate estimate is given by figure 13 . The two turns are well identified with a value of about $+/-1.3$ degree/sec in turns (it must be noticed that the ground turning rate is different from the air turning rate). The results of this approach are

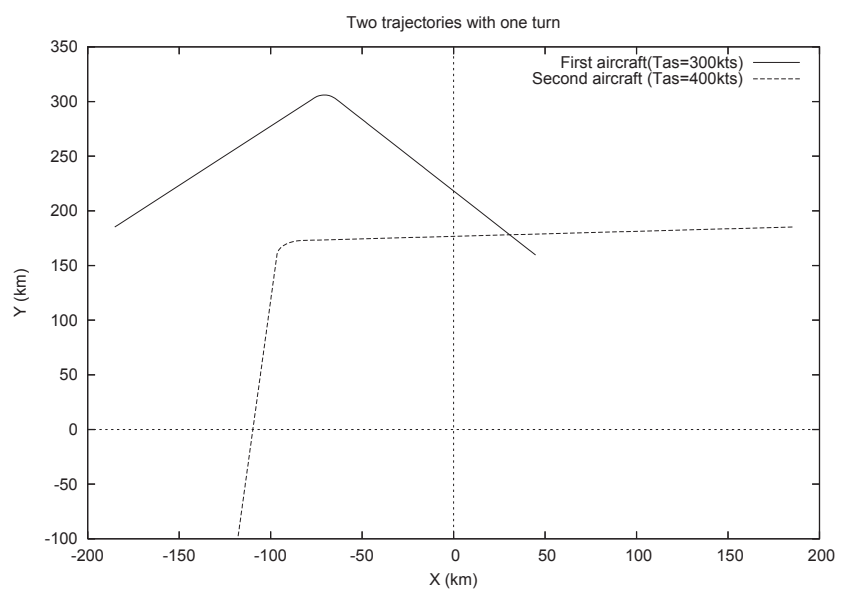

Figure 14. Second trajectory set. Each straight lines 1 ast 20 minutes. The turning rate in the first trajectory is $1 \mathrm{deg}$ and $-1 \mathrm{deg}$ for the second one. The true air spee of the first aircraft is $300 \mathrm{kts}$ and 400 for the second one

given in the following table :

\begin{tabular}{|c|c|c|}
\hline $\begin{array}{c}\text { Wind } \\
\text { Est }\end{array}$ & $\begin{array}{c}\text { Strength } \\
\text { and Angle }\end{array}$ & Error \\
\hline$w_{x}=-17.6798 \mathrm{~m} / \mathrm{s}$ & $39.65 \mathrm{kts}$ & $0.35 \mathrm{kts}$ \\
$w_{y}=-10.1831 \mathrm{~m} / \mathrm{s}$ & $240.053 \mathrm{deg}$ & $0.053 \mathrm{deg}$ \\
\hline
\end{tabular}

Those results has been computed by using equations (7), (8) and the following ground speed estimates coming from the averaging process :

$$
\begin{gathered}
v_{1 x}=54.4818 \mathrm{~m} / \mathrm{s} v_{1 y}=61.9523 \mathrm{~m} / \mathrm{s} \\
v_{2 x}=84.3536 \mathrm{~m} / \mathrm{s} v_{2 y}=-10.2142 \mathrm{~m} / \mathrm{s} \\
v_{3 x}=-17.6780 \mathrm{~m} / \mathrm{s} v_{3 y}=91.8504 \mathrm{~m} / \mathrm{s}
\end{gathered}
$$

When two aircraft trajectories are available, only one turn for each is necessary. For such a model, the trajectories given on figure 14 has been used. The turning rate estimates are given on figures 15 and 16 . The results of this approach are given in the following table :

\begin{tabular}{|c|c|c|}
\hline $\begin{array}{c}\text { Wind } \\
\text { Est }\end{array}$ & $\begin{array}{c}\text { Strength } \\
\text { Angle }\end{array}$ & Error \\
\hline$w_{x}=-17.6485 \mathrm{~m} / \mathrm{s}$ & $39.64 \mathrm{kts}$ & $0.36 \mathrm{kts}$ \\
$w_{y}=-10.2227 \mathrm{~m} / \mathrm{s}$ & $239.918 \mathrm{deg}$ & $0.082 \mathrm{deg}$ \\
\hline
\end{tabular}

Those results has been computed by the using equations 1213 and the ground speed estimates coming from the averaging process :

$v a_{1 x}=90.5494 \mathrm{~m} / \mathrm{s} v a_{1 y}=98.0082 \mathrm{~m} / \mathrm{s}$
$v a_{2 x}=90.5552 \mathrm{~m} / \mathrm{s} v a_{2 y}=-118.4478 \mathrm{~m} / \mathrm{s}$
$v b_{1 x}=-221.7254 \mathrm{~m} / \mathrm{s} v b_{1 y}=-10.2111 \mathrm{~m} / \mathrm{s}$
$v b_{2 x}=-17.6796 \mathrm{~m} / \mathrm{s} v b_{2 y}=-214.2995 \mathrm{~m} / \mathrm{s}$




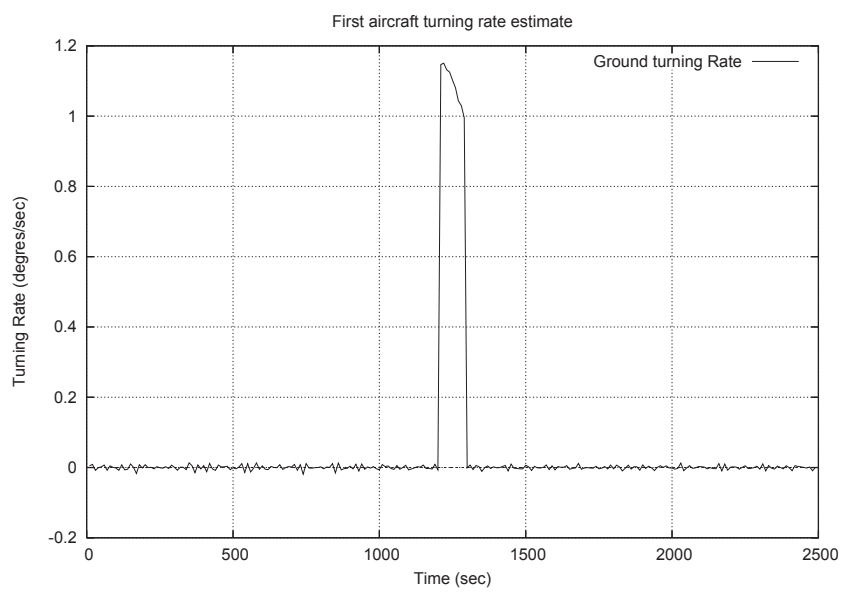

Figure 15. Turning Rate Estimate of the First Aircraft

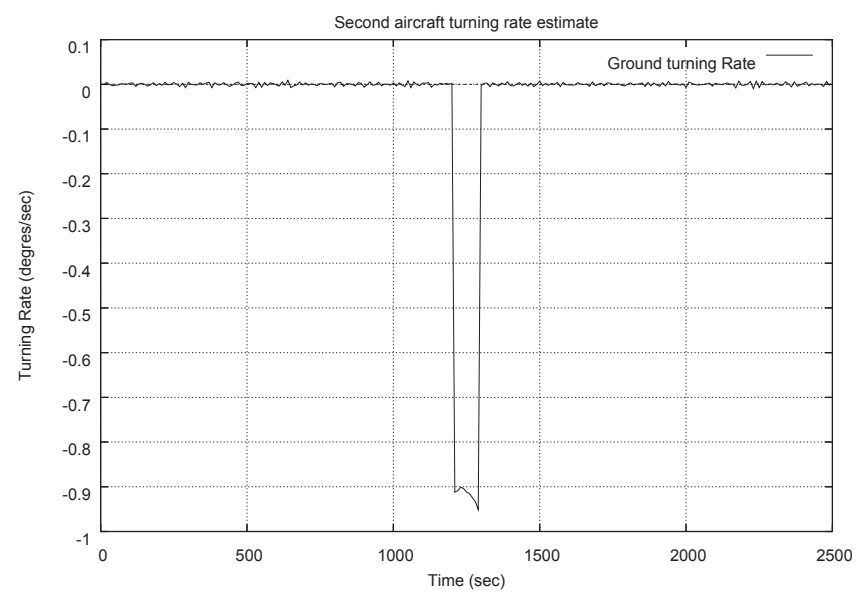

Figure 16. Turning Rate Estimate of the Second Aircraft

Having now some wind estimates on some points in the airspace (where the aircraft are located), the next step consists in the global wind field interpolation based on the meteorological model called "ShallowWater". This model is described in [6] and the associated discret expansion in given in [17].

\section{Vector Spline Interpolation}

\section{Shallow Water Wind Model}

Let $V$ be the vector field giving the wind velocity at each point. We will assume in the following that area of interest is located high enough above the ground level so that viscous effects are negligible. Assume furthermore that wind field is two dimensional (that is does not depends on altitude). Then the evolution of $V$ is described by the system of equations :

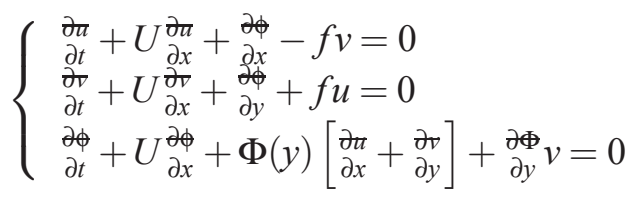

with :

$$
V=\left[\begin{array}{c}
U+u \\
v
\end{array}\right]
$$

$U$ the mean west-east component of the wind, $f$ is the Coriolis parameter which can be expressed as :

$$
f=2 \Omega \sin \phi_{0}-2 \Omega R^{-1} \cos \phi_{0}
$$

with $\Omega=\frac{2 \pi}{86400}$ the rotation rate of the earth, $\phi_{0}$ the latitude (assumed to be constant in the domain of interest) and $R=6.370 \times 10^{6} \mathrm{~m}$ the earth radius. $\Phi$ is the geopotential of the isobaric surface and is written as :

$$
\Phi=\theta(y)+\phi
$$

with :

$$
\theta(y)=\theta_{0}-\left[2 \Omega\left(\sin \phi_{0}\right) y-\Omega R^{-1}\left(\cos \phi_{0}\right) y^{2}\right] U
$$

and :

$$
\phi_{0}=3 \times 10^{4} \mathrm{~ms}^{-2} \quad U=25 \mathrm{~ms}^{-1}
$$

The set of equations 44 is known as shallow water model. With little rewriting, it can be shown that this model has the following synthetic form :

$$
\left(\frac{\partial}{\partial t}+L\right) X=0
$$

with :

$$
X=\left[\begin{array}{l}
u \\
v \\
\phi
\end{array}\right]
$$

and $L$ the differential operator :

$$
L=A \frac{\partial}{\partial X}+B \frac{\partial}{\partial y}+C
$$

where

$$
\begin{gathered}
A=\left[\begin{array}{ccc}
U & 0 & 0 \\
0 & U & 0 \\
\Phi & 0 & U
\end{array}\right] \quad B=\left[\begin{array}{lll}
0 & 0 & 0 \\
0 & 0 & 1 \\
0 & \Phi & 0
\end{array}\right] \\
C=\left[\begin{array}{ccc}
0 & -f & 0 \\
f & 0 & 0 \\
0 & \Phi^{\prime} & 0
\end{array}\right]
\end{gathered}
$$

Interpolating Vector Splines 
The symbol $\sigma(y, \xi)$ of this differential operator $L$ easily computes as :

$\sigma(y, \xi)=\left[\begin{array}{ccc}-i \xi_{1} U & -f & 0 \\ f & -i \xi_{1} U & -i \xi_{2} \\ -i \xi_{1} \Phi(y) & -i \xi_{2} \Phi(y)+\Phi^{\prime}(y) & -i \xi_{1} U\end{array}\right]$

Since a solution 0 of the Shallow-Water model must fulfill :

$$
\left(\frac{\partial}{\partial t}+L\right) V=0
$$

we will seek for a vector field $V$ such that :

$$
V=\operatorname{argmin} \int_{0}^{T} \int_{D}\left\|\left(\frac{\partial}{\partial t}+L\right) X(x, t)\right\|^{2} d x d t
$$

under the constraints that :

$$
X\left(x_{i}, t_{i}\right)=v_{i}, i=1 \ldots n
$$

if $v_{i}$ is the wind measurement at time $t_{i}$ and location $x_{i}, D$ is the domain of interest and $I$ is the time interval chosen for computation. Assuming that boundary conditions vanish, we obtain that the functional criterion has expression :

$$
\int_{I} \int_{D}\left\langle\left(-\frac{\partial^{2}}{\partial t^{2}}+L^{t} L\right) X(x, t), X(x, t)\right\rangle d x d t
$$

with $L^{t}$ the adjoint operator of operator $L$. Operator $L^{t} L$ has symbol :

$\sigma(y, \xi)=\left[\begin{array}{ccc}\xi_{1}^{2}\left(\Phi^{2}+U^{2}\right)+f^{2} & \xi_{1} \xi_{2} \Phi+i \xi_{1}\left(\Phi \Phi^{\prime}-2 f U\right) & \left.-i \xi_{1}+\xi_{2} U \Phi\right) \\ \xi_{1} \xi_{2} \Phi-i \xi_{1}\left(\Phi \Phi^{\prime}-2 f U\right. & f^{2}+\xi_{1}^{2} U^{2}+\xi_{2}^{2} \Phi^{2}+\Phi^{\prime 2} & \xi_{1} \xi_{2}(U+\Phi)-i \xi_{2} \Phi^{\prime} \\ i \xi_{1}+\xi_{2} U \Phi & \xi_{1} \xi_{2}(U+\Phi)+i \xi_{2} \Phi^{\prime} & \xi_{1}^{2}\end{array}\right]$

$L^{t} L$ admits an eigenvector expansion $\left(\phi_{n}\right)_{n \in \mathbb{N}}$ with associated eigenvalues $\left(\lambda_{n}\right)_{n \in \mathbb{N}}$. We will week for a kernel $K(x, y, t, u)$ such that :

$$
\begin{aligned}
& \int_{I} \int_{D}\langle K(x, y, t, u),\left.\left(-\frac{\partial^{2}}{\partial t^{2}}+L^{t} L\right) X(y, u)\right\rangle d y d u \\
&=X(x, t)
\end{aligned}
$$

Formally, we may write :

$$
K(x, y, t, u)=\sum_{n} c_{n}(t, u) \phi_{n}(x) \otimes \phi_{n}(y)
$$

and find the right expression for $c_{n}(t, u)$. In the case $I=\mathbb{R}$ and wind fields of finite energy over time, some computations show that the sought after kernel has expression :

$$
K(x, y, t, u)=\sum_{n} \frac{e^{-\mu_{n}|t-u|}}{2 \mu_{n}} \phi_{n}(x) \otimes \phi_{n}(y)
$$

with : $\mu_{n}=\sqrt{\lambda_{n}}$. The constraints that $X\left(x_{i}, t_{i}\right)=v_{i}, i=$ $1 \ldots n$ may thus be rewritten as :

$$
\int_{I} \int_{D}\left\langle K\left(x_{i}, y, t_{i}, u\right),\left(-\frac{\partial^{2}}{\partial t^{2}}+L^{t} L\right) X(y, u)\right\rangle d y d u=v_{i}
$$

which is much more tractable than the point version. Introducing Lagrange multipliers $\left(\varepsilon_{i}\right)_{i=1 \ldots n}$, the problem may be solved and the solution written as :

$$
X(x, t)=\sum_{i=1 \ldots n} \varepsilon_{i} K\left(x_{i}, x, t_{i}, t\right)
$$

so that optimal vector field is a linear combination of kernels evaluated at measurement points $\left(x_{i}, t_{i}\right)_{i=1 \ldots n}$. This is a classical result within the frame of interpolating spline theory, with the restriction that the kernel is not translation invariant. From now, only numeric evaluations of the kernel can be made, based on finding finite elements approximations of the eigenfunctions, then truncating the infinite sum in the kernel expansion at an order where residual is low enough. This yields a piecewise polynomial approximate kernel, which can be computed once and used afterward (note that if the kernel is computable, finding the wind field expansion is done by solving a linear system).

\section{Conclusion}

This paper has given a new approach for extracting the wind information from the radar tracks. Two approaches has been presented. When True Airspeed measures are available, linear models may be used with regular Kalman filter. In this first approach, wind estimate are available all along the aircraft trajectory. When only position measures are available, an observability analysis has shown that wind may be estimated only after turns (one turn for two aircraft or two turns for one aircraft). For such approach, a closed form of the wind has been developed. Then, those models have been validated with realistic simulations. Having wind measures spread in the airspace, an interpolating method using vector splines has been developed. Based on the differential operator of the Sallow-Water model and the local wind observations, this method build a vector field using kernel functions. The associated eigenfunctions $\left(\phi_{n}\right)$ have to be computed numerically on each point of the grid where the vector field has to be computed. It must be noticed that the Shallow-Water is only valid above $2000 \mathrm{~m}$; this means this method may be used mainly for En-Route traffic. 


\section{References}

[1] B.D.O. Anderson and J.B. Moore. Optimal Filtering. Prentice Hall, 1979.

[2] R.B. Asher, P.S. Maybeck, and R.A.K. Mitchell. Filtering for precision pointing and tracking with application for aircraft to satellite tracking. In Proceedings IEEE Conference Decision and Control. IEEE, 1975.

[3] R.H. Battin. Astraunotical Guidance. McGraw-Hill, 1964.

[4] Bradford. Using aircraft radar tracks to estimate winds aloft. The Lincoln Laboratory Journal, 2, 1989.

[5] C.K. Chui and G Chen. Kalman Filtering with Real-Time Applications. Springer-Verlag, 1987.

[6] S.E Cohn and T.F Parrish. The behavior of the forcast error covariances for a kalman filter in two dimensions. Monthly Weather Review, 119:1757-1785, 1991.

[7] R.E et al. Cole. Wind prediction accuracy for air traffic management decision support tools. In Proceedings of $3^{\text {th }}$ USA-Europe ATM Seminar. FAA-Eurocontrol, 2000.

[8] D Delahaye. Wind field update using radar track data. Master's thesis, Ecole Nationale de l'Aviation Civile, 1992.

[9] $\mathrm{H}$ et al. Erzberger. Conflict detection and resolution in the presence of prediction error. In Proceedings of $1^{\text {th }}$ USA-Europe ATM Seminar. FAA-Eurocontrol, 1997.

[10] S. Grewal, Mohinder and Andrews Angus P. Kalman Filtering Theory and Practice. Prentice Hall, 1993.

[11] Simon J. Julier and Jeffery K. Uhlmann. A new extension of the kalman filter to nonlinear systems.
In Proceedings of AeroSense: The 11th International Symposium on Aerospace/Defense Sensing, Simulation and Controls, Multi Sensor Fusion, Tracking and Resource Management II. SPIE, 1997.

[12] R.E Kalman. A new approach to linear filtering and prediction problems. Transactions of the ASME-Journal of Basic Engineering, 82(Series D):35-45, 1960.

[13] C.C Lefas. Improved tracking with mode-s data-linked velocity measurements. IEEE Transactions on Aerospace and Electronic Systems, 27(4):709-714, 1991.

[14] P.S. Maybeck. Stochastic Models, Estimation, and Control (vol 1 and 2). Academic Press, 1982.

[15] S Mondoloni and D Liang. Improving trajectory forecasting through adaptive filtering technique. In Proceedings of $5^{\text {th }}$ USA-Europe ATM Seminar. FAA-Eurocontrol, 2003.

[16] C.M Rekkas, C.C Lefas, and N.J Krikelis. Three dimensional tracking using on-board measurements. IEEE Transactions on Aerospace and Electronic Systems, 27(4):617- 624, 1991.

[17] R.D Richtmyer and K.W Morton. Difference Methods for Initial-Value Problems. Krieger Publishing Company, 1994.

[18] G. Welch and G Bishop. An introduction to the kalman filter. Technical report, University of North Carolina. Department of Computer Science, 1995.

\section{Email Addresses}

Daniel Delahaye: delahaye@recherche.enac.fr

StephanePuechmorel: puechmor@recherche.enac.fr 


\section{Appendix A : Unscented Kalman Filter}

The unscented Kalman filter (UKF) [11] uses a deterministic sampling technique known as the unscented transform to pick a minimal set of sample points (called sigma points) around the mean. These sigma points are then propagated through the nonlinear functions and the covariance of the estimate is then recovered. The result is a filter which more accurately captures the true mean and covariance. In addition, this technique removes the requirement to analytically calculate Jacobians, which for complex functions can be a difficult task in itself.

\section{Prediction}

As with the EKF, the UKF prediction can be used independently from the UKF update.

The estimated state and covariance are augmented with the mean and covariance of the process noise.

$$
\begin{aligned}
& \underline{X}_{k-1 \mid k-1}^{a}=\left[\begin{array}{ll}
\hat{X}_{k-1 \mid k-1}^{T} & E\left[\underline{v}_{k}^{T}\right]
\end{array}\right]^{T} \\
& \underline{P}_{k-1 \mid k-1}^{a}=\left[\begin{array}{cc}
P_{k-1 \mid k-1} & 0 \\
0 & \underline{Q}_{k}
\end{array}\right]
\end{aligned}
$$

A set of $2 L+1$ sigma points is derived from the augmented state and covariance where $L$ is the dimension of the augmented state.

$$
\begin{aligned}
\underline{\chi}_{k-1 \mid k-1}^{0}= & \underline{X}_{k-1 \mid k-1}^{a} \\
\underline{\chi}_{k-1 \mid k-1}^{i}= & \underline{X}_{k-1 \mid k-1}^{a}+\left(\sqrt{(L+\lambda) \underline{P}_{k-1 \mid k-1}^{a}}\right)_{i} \\
& i=1 . . L \\
\underline{\chi}_{k-1 \mid k-1}^{i}= & \underline{X}_{k-1 \mid k-1}^{a}-\left(\sqrt{(L+\lambda) \underline{P}_{k-1 \mid k-1}^{a}}\right)_{i-L} \\
& i=L+1, \ldots 2 L
\end{aligned}
$$

where

$$
\left(\sqrt{(L+\lambda) \underline{P}_{k-1 \mid k-1}^{a}}\right)_{i}
$$

is the $i$ th column of the matrix square root of

$$
(L+\lambda) \underline{P}_{k-1 \mid k-1}^{a}
$$
satisfies

using the definition: square $\operatorname{root} A$ of matrix $B$

$$
B \equiv A A^{T}
$$

The matrix square root should be calculated using numerically efficient and stable methods such as the Cholesky decomposition. The sigma points are propagated through the transition function $\underline{\mathcal{F}}$.

$$
\underline{\chi}_{k \mid k-1}^{i}=\underline{\mathcal{F}}\left(\underline{\chi}_{k-1 \mid k-1}^{i}\right) \quad i=0 . .2 L
$$

The weighted sigma points are recombined to produce the predicted state and covariance.

$$
\begin{gathered}
\underline{\hat{X}}_{k \mid k-1}=\sum_{i=0}^{2 L} W_{s}^{i} \underline{\chi}_{k \mid k-1}^{i} \\
\left.\underline{P}_{k \mid k-1}=\sum_{i=0}^{2 L} W_{c}^{i}\left[\underline{\chi}_{k \mid k-1}^{i}-\underline{\hat{X}}_{k \mid k-1}\right] \underline{\chi}_{k \mid k-1}^{i}-\underline{\hat{X}}_{k \mid k-1}\right]^{T}
\end{gathered}
$$

where the weights for the state and covariance are given by:

$$
\begin{gathered}
W_{s}^{0}=\frac{\lambda}{L+\lambda} \\
W_{c}^{0}=\frac{\lambda}{L+\lambda}+\left(1-\alpha^{2}+\beta\right) \\
W_{s}^{i}=W_{c}^{i}=\frac{1}{2(L+\lambda)} \\
\lambda=\alpha^{2}(L+\kappa)-L
\end{gathered}
$$

Typical values for $\alpha, \beta$ and $\kappa$ are $10^{-3}, 2$ and 0 respectively.

\section{Update}

The predicted state and covariance are augmented as before, except now with the mean and covariance of the measurement noise.

$$
\begin{gathered}
\underline{X}_{k \mid k-1}^{a}=\left[\begin{array}{ll}
\hat{X}_{k \mid k-1}^{T} & E\left[\underline{w}_{k}^{T}\right]
\end{array}\right]^{T} \\
\underline{P}_{k \mid k-1}^{a}=\left[\begin{array}{cc}
\underline{P}_{k \mid k-1} & 0 \\
0 & \underline{R}_{k}
\end{array}\right]
\end{gathered}
$$

As before, a set of $2 L+1$ sigma points is derived from the augmented state and covariance where $L$ is the dimension of the augmented state.

$$
\begin{aligned}
\underline{\chi}_{k \mid k-1}^{0}= & \underline{X}_{k \mid k-1}^{a} \\
\underline{\chi}_{k \mid k-1}^{i}= & \underline{X}_{k \mid k-1}^{a}+\left(\sqrt{(L+\lambda) \underline{P}_{k \mid k-1}^{a}}\right)_{i} \\
& i=1 . . L \\
\underline{\chi}_{k \mid k-1}^{i}= & \underline{X}_{k \mid k-1}^{a}-\left(\sqrt{(L+\lambda) \underline{P}_{k \mid k-1}^{a}}\right)_{i-L} \\
& i=L+1, \ldots 2 L
\end{aligned}
$$


Alternatively if the UKF prediction has been used the sigma points themselves can be augmented along the following lines

$$
\underline{\chi}_{k \mid k-1}:=\left[\underline{\chi}_{k \mid k-1}^{T} \quad E\left[\underline{w}_{k}^{T}\right]\right]^{T} \pm \sqrt{(L+\lambda) \underline{R}_{k}^{a}}
$$

where

$$
\underline{R}_{k}^{a}=\left[\begin{array}{cc}
0 & 0 \\
0 & \underline{R}_{k}
\end{array}\right]
$$

The sigma points are projected through the observation function $\underline{\mathcal{H}}$.

$$
\underline{\gamma}_{k}^{i}=\underline{\mathcal{H}}\left(\underline{\chi}_{k \mid k-1}^{i}\right) \quad i=0 . .2 L
$$

The weighted sigma points are recombined to produce the predicted measurement and predicted measurement covariance.

$$
\begin{gathered}
\underline{\hat{Z}}_{k}=\sum_{i=0}^{2 L} W_{s}^{i} \underline{\gamma}_{k}^{i} \\
\underline{P}_{z_{k} z_{k}}=\sum_{i=0}^{2 L} W_{c}^{i}\left[\underline{\gamma}_{k}^{i}-\underline{\hat{Z}}_{k}\right]\left[\underline{\gamma}_{k}^{i}-\underline{\hat{Z}}_{k}\right]^{T}
\end{gathered}
$$

The state-measurement cross-covariance matrix,

$$
\underline{P}_{x_{k} z_{k}}=\sum_{i=0}^{2 L} W_{c}^{i}\left[\underline{\chi}_{k \mid k-1}^{i}-\underline{\hat{X}}_{k \mid k-1}\right]\left[\underline{\gamma}_{k}^{i}-\underline{\hat{Z}}_{k}\right]^{T}
$$

is used to compute the UKF Kalman gain.

$$
K_{k}=\underline{P}_{x_{k} z_{k}} \underline{P}_{z_{k} z_{k}}^{-1}
$$

As with the Kalman filter, the updated state is the predicted state plus the innovation weighted by the Kalman gain,

$$
\underline{\hat{X}}_{k \mid k}=\underline{\hat{X}}_{k \mid k-1}+K_{k}\left(\underline{Z}_{k}-\underline{\hat{Z}}_{k}\right)
$$

And the updated covariance is the predicted covariance, minus the predicted measurement covariance, weighted by the Kalman gain.

$$
\underline{P}_{k \mid k}=\underline{P}_{k \mid k-1}-K_{k}{\underline{P_{z_{k} z_{k}}}}_{K_{k}^{T}}^{T}
$$

